\title{
Testing the Effects of Micro-Pulse Stimulation on Blood Circulation Using the Thermodynamic Sensors
}

Marie Tobolova ${ }^{1 *}$, Milan Chmelar ${ }^{1}$, Ivo Provaznik ${ }^{1}$, Zdenek Reznicek ${ }^{2}$, Radovan Kabes ${ }^{3}$ and Jaromir Bastinec $^{4}$

${ }^{1}$ Department of Biomedical Engineering, Faculty of Electrical Engineering and Communication, Brno University of Technology, Czech Republic

${ }^{2}$ Hybrid Integrated Technologies Ltd., Czech Republic

${ }^{3}$ Equine Clinic, Faculty of Veterinary Medicine, University of Veterinary and Pharmaceutical Sciences Brno, Czech Republic

${ }^{4}$ Department of Mathematics, Faculty of Electrical Engineering and Communication, Brno University of Technology, Czech Republic

\section{Abstract}

Reasons for performing study: Electrotherapy is used in human medicine and successively also in veterinary practice, but relatively little is known about the mechanisms of action in detail.This study is focused on identifying the physiological effects of micro-pulse stimulation, a new method designed for equine electrotherapy, using the thermodynamic sensing.

Objectives: To establish on the basis of measurement with the Thermo Dynamic Sensors, if the micro-pulse stimulation has any effect on blood circulation.

Methods: Twenty horses participated in this study. Two miniature Thermo Dynamic Sensors (TDS), working on the principle of the balance energy equilibrium and special designed for the measurement on horses, were placed on both forelimbs and monitored the changes in thermal activity. Micro-pulse stimulation of specified parameters was applied for a defined time only on one limb and the other was just considered as a reference. The measured responses were statistically processed, compared and evaluated.

Results: The measured data were processed by basic and multivariate statistical analysis (correlation, Wilcoxon test, multidimensional scaling, and cluster analysis) which identified a significant difference between signals from stimulated and reference limb. A considerable increase of thermal activity is evident in signals from stimulated limb during the applied micro-pulse stimulation.

Conclusions: Based on the results, it seems the micro-pulse stimulation really has physiological effect consisting in an increased blood perfusion which is associated with the warming-up of the stimulated area and this is detectable by the thermodynamic sensor.

Potential relevance: Comparison and statistical evaluation of the measured signals provided a more detailed view on the thermal changes within the stimulated area, which is significantly related to blood circulation in limbs, and also with the support of the reduction of edema which could be one of the practical applications of micro-pulse stimulation (also tested parallel to this study).

Keywords: Micro-pulse stimulation; Thermodynamic sensor; Blood circulation measuring; Horse

\section{Introduction}

The aim of this study is to analyse if the micro-pulse stimulation influences the blood circulation. An important part of this lies in finding an objective way how to investigate it. In general, the blood circulation could be measured by various methods but not all are of the same reliability and also patient-friendly. Most techniques are based on an indirect measurement [1], such as impedance pletysmography or Doppler ultrasound method. The great advantage of them lies in their non-invasive character and minimum discomfort for the patient but to the prejudice of accuracy and responsibility of the results. There also take place the thermodynamic methods based on a skin temperature measuring.

Usually, the thermometers working on the thermocouple or resistance thermometer principle are used. The detectable temperature value is the basic physical magnitude for a description and monitoring the thermodynamic system state and it is a result of the heat inflow into the subcutaneous tissue and the ambient heat release. The temperature is a state value and gives objective information only in case this state is stable [2]. The system stabilization does not establish immediately, but it is a consequence of the previous changes of the system activity. Therefore, there are some doubts about the method accuracy because the bloodstream is definitively a dynamic system and its stability of remaining in one state after a few seconds is really improbable. The blood circulates in the vessels continuously with respect to the heart pumping activity. It seems more beneficial to monitor the thermal activity changes of the system directly as a cause of the temperature ratio changes in the system leading to the new balance equilibrium state establishment $[2,3]$. This resolution offers the innovative approach of the thermodynamic sensing.

\section{Materials and Methods}

\section{Instrumentation}

Microstimulator was designed as a small, portable, batteryoperated device easy to use and suitable for the animal electrotherapy,

*Corresponding author: Marie Tobolova, Department of Biomedical Engineering, Faculty of Electrical Engineering and Communication, Brno University of Technology, Czech Republic, Tel: +420 541146 6; E-mail: tobolovam@gmail.com

Received January 11, 2014; Accepted February 07, 2014; Published February 14,2014

Citation: Tobolova M, Chmelar M, Provaznik I, Reznicek Z, Kabes R, et al (2014) Testing the Effects of Micro-Pulse Stimulation on Blood Circulation Using the Thermodynamic Sensors. J Biosens Bioelectron 5: 147. doi: 10.4172/21556210.1000147

Copyright: (c 2014 Tobolova M, et al. This is an open-access article distributed under the terms of the Creative Commons Attribution License, which permits unrestricted use, distribution, and reproduction in any medium, provided the original author and source are credited. 
particularly for horses that were the target objects for the micro-pulse stimulation testing [4,5]. It generates high-voltage pulses (about hundreds volts) with a very short duration (microseconds). The device is classified as a medical electrical device and the strict requirements for the design and function according to the relevant standards were kept. Based on the executed tests of the accredited testing laboratory, it carries the international CE mark.

For monitoring the fast-changing thermal activity in the limb during the micro-pulse stimulation, the special thermodynamic sensors were designed and manufactured by the specialized company. These, so-called resistive Thermo Dynamic Sensors (TDS), work on the principle of the balance energy equilibrium. An intention of the authors was to integrate into the real thermodynamic system a unique element monitoring both the steady state and non-steady state [6]. This way of sensing, as so far the only one, makes possible an evaluation of the minor temperature differences close to zero and enables complex monitoring of the energy activity in any thermodynamic system that is not possible by classic methods said above. Thanks to its extraordinary sensitivity, these sensors have found place in variety of industrial, electrochemical, biotechnological and nano-applications [7].

\section{Experimental design}

A schematic diagram of the whole measuring system shows Figure 1 and the real measurement is shown at Figure 2 (right). The experiment is designed as a parallel measurement of the signals of both forelimbs using the TDS when only one limb is stimulated whereas the second one is considered as a reference. The miniature TDS is fastened by a band at each forelimb so that it has a full contact with the skin without an uncomfortable pressure. The connection of the TDS and external thermodynamic part is formed by an extension cord terminating with the easy-disconnecting connectors, which is a safety precaution for the fast disconnection in case of an unexpected action of the horse and a prevention of the cable breaking and sensor damage.

The thermal activity changes monitored by TDS are converted to the electric signal which is measurable by the connected digital multimeters equipped with an optical USB interface for a communication with the notebook. A two-channel recording was realized in the console of the Linux OS using the special programme designed for this application.
The TDS position at the limb was chosen for the following reasons. Although the well perfused region is particularly the lower part of the limb, closer to the hoof, the TDS was placed just above the horses' knee Figure 2 (left). It is a place where some main blood vessels and nerves of the forelimb pass, particularly arteria mediana, vena mediana, vena cephalica, nervus medianus, nervus cutaneous antebrachii medialis and nervusradialis [8]. The veins are palpable and even visible by naked eye in some cases, especially after the hard work.

It is quite unfeasible to place the TDS in the lower part of the limb because of the cable connections TDS with the external thermodynamic part. Any slightest move of the limb causes a radius much greater than in the arm. Therefore, it can lead to the abortive disconnection of the cables and constraint of the running monitoring. It is necessary to take these similar situations into consideration because the horse is sensible and shy. Usually, it perceives very intensively each stimulus, unknown noise and surrounding changes in general. There was an effort to avoid the horse nervousness which would surely subscribe to whole measurement in a negative way. But it is not possible to keep fully equably conditions for the whole time of monitoring because an activity in the stable could be influenced really hard, as well as the horses' reaction on it. Therefore, number of artefacts and measuring faults, that cannot be affected, could occur.

The TDS were placed at the cleaned forelimbs as the first, and then the extension cords were connected between the TDS and the external unit. Consequently, the whole apparatus was turned on. The sensors need some time for stabilization and adaptation to the skin temperature. But it is not possible to expect stagnancy at the constant value because the processes in the limb have a dynamic character. After the sensors' stabilization, that can be characterized as an oscillation of the measured values around any average value and no sudden fluctuations, the proper monitoring could start. The output of the programme (signals from reference and stimulated limbs in each time step) was displayed online. The electrodes of the working microstimulator were placed in the distance of about 5 centimetres above the fastened sensor at the limb, assigned as stimulated, for the period of 30 seconds. After this period, the stimulation was broken off and microstimulator was removed, but the monitoring continued approximately next 130 seconds. A short resting pause followed the measurement for rest of the horse and for

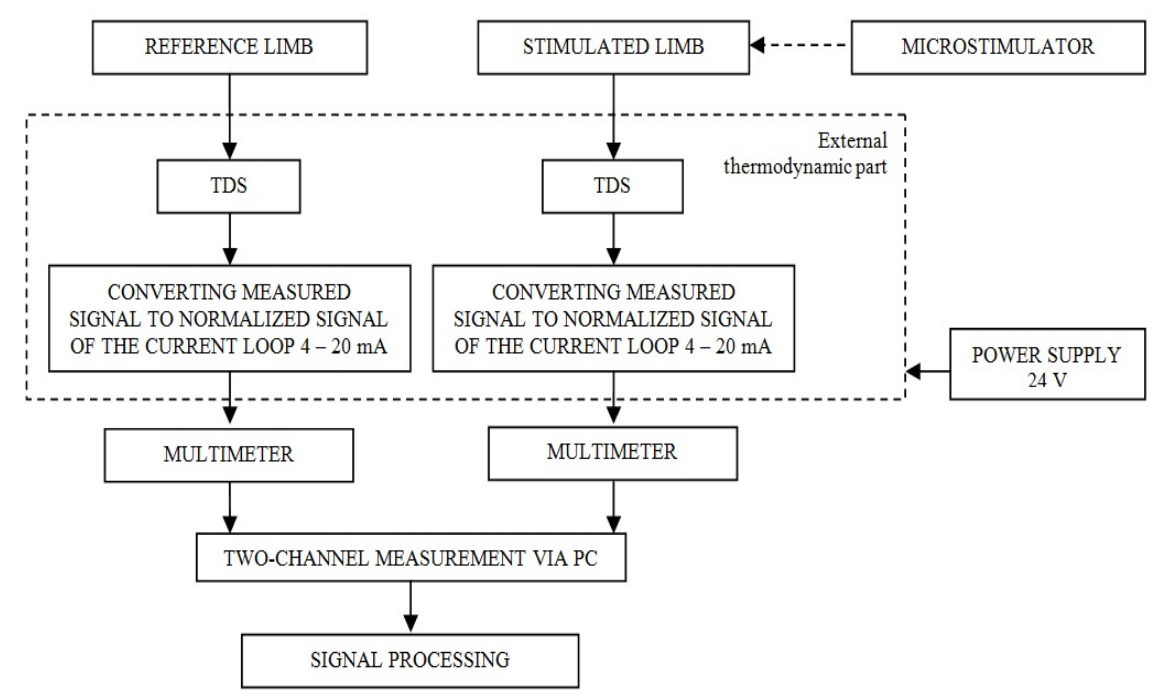

Figure 1: The schematic diagram of the experiment design [5] 


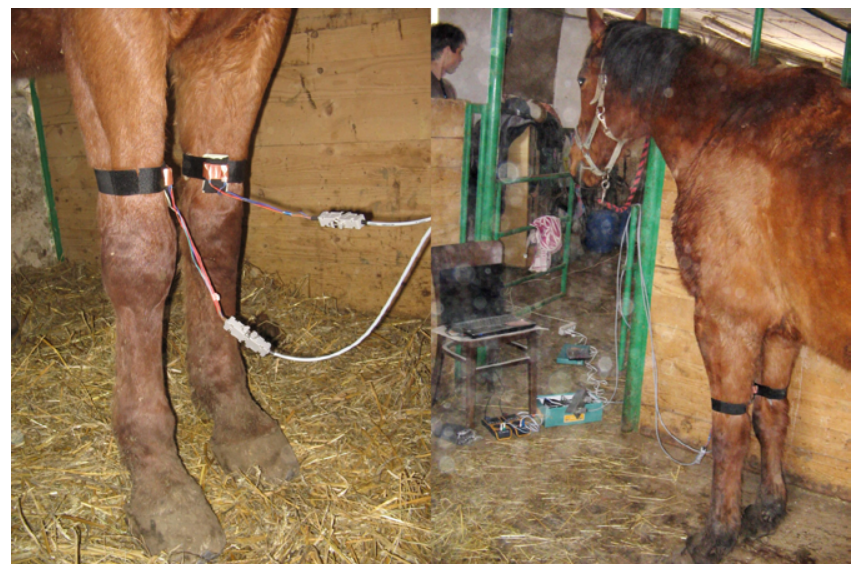

Figure 2: Left: TDS position on the limb in detail. Right: The measuring apparatus in practice [5]

the stabilization of the TDS before next repeating measurement with different parameters of the stimulation.

Overall, four measurements for four regimes of stimulation were applied on each horse. It concerned the combinations of minimal and maximal levels of intensity and frequency. Particular regimes were signed by an alpha-numeric code $\mathrm{Rxx}$, where $\mathrm{x}=0 / 1$. The first number of the code expresses an intensity setting and the second relates to the frequency. Altogether, four possible combinations of stimulation were set in and applied in the following order:

1) R00-min intensity, min frequency

2) R01-min intensity, max frequency

3) R10-max intensity, min frequency

4) R11-max intensity, max frequency

It was necessary to optimize the monitoring time to the real conditions. An ideal conception is definitely to carry out the measurement as long as possible, for example in order of tens of minutes, which could best distinguish the processes caused by the stimulation from those in the background. But it is almost impossible to make the horse stay absolutely motionless during the time of monitoring. The situation is quite different from the laboratory animals because this problem is solved by using anaesthesia. But it was definitely excluded in this experiment. Therefore, it was necessary to choose a compromise between the time when the horse is disposed to stop still and the time that is needed for the data acquisition with the best predicative value as possible.

\section{Animal's selection}

The experiment ran in period from February to March for four weeks. All data were kept anonymous and the alpha-numeric code Kxx was assigned to each horse. The adult horses without the sex limitations (mares, stallions and geldings) between the ages of 5 and 22 were included. For the best possible homogeneity of the selected population, individuals of the Czech Warmblood Horse breed were chosen in preference. Nevertheless, no less important criterion of the horse participation in the experiment was its balanced character and goodwill to stand still for the monitoring time, which is unfortunately not common for a number of horses. The Czech Warm blood Horse breed forms a large group of 15 individuals, then three Czech Warm blood Horse half-breds and two English Thoroughbreds. This selection is explained in connection with the history of breeding $[9,10]$. From this perspective, the Czech Warmblood is a result of the crossbreeding of several types of horse and it is not a pure breed at all, such as the Thoroughbred that has only a few common grandsires. On the other hand, the Warmblood still contains a high part just of the Thoroughbred blood. Therefore, the experiment would not be negative influenced by the participating half-breds and thorough breds. Conversely, this supported a variability of the tested population.

\section{Data analysis}

The aim of the whole experiment is to find the answer, if the micro-pulse stimulation causes any physiological effect on the bloodstream which evokes a change in the thermal energy distribution of the stimulated area detectable by the thermodynamic sensors. The statistical analysis of the measured reference and stimulated limbs' signals proposed to answer the following questions:

(A) Is there a significant difference between the signals from reference and stimulated limb that would point at the apparent effect of the micro-pulse stimulation on the blood distribution in the bloodstream and thermal changes connected with it?

(B) If the difference exists at particular measurements, can we expect some similarities of all reference or stimulated signals among horses in all measurements?

The first information about the character of the measured data was possible to obtain after their XY diagram depiction, thus the time dependence of TDS responses from reference and stimulated limbs at each horse. An example of that diagram is shown in Figure 3. It is a comparison of the signals measured at two horses (K01, K02). The overwhelming majority of stimulated signals had common relatively fast growth during the stimulation period whereas the reference signals had entirely stochastic character. Therefore, it seems the stimulation influences the intrinsic environment of the stimulated area and this change appears in the TDS signal. Although this information is not sufficient for an objective consideration of the data, it presents the basic conception of the data, if there is some worth information for more detailed investigation and evaluation. The graphical comparison of the reference (further only an abbreviation REF) and stimulated signals (further an abbreviation STIM) showed a relative discrepancy but this claim was not substantiated and it was necessary to verify its reliability by the statistical tests.

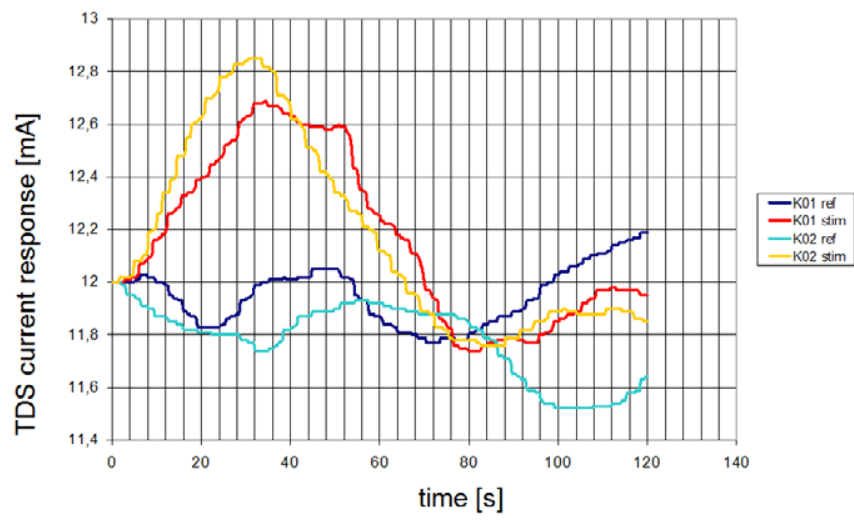

Figure 3: Comparison of the reference (ref) and stimulated (stim) signals measured at two horses $(\mathrm{K} 01, \mathrm{~K} 02)$ in the R00 regime of stimulation [5] 
It was practically impossible to find simple description characteristics of all measurements' distribution. A number of them showed bimodality, various types of skewness and extreme values [5]. But there was needed a circumspection, because the extreme and outlying values are usually understood as something unfavourable and a lot of software try to suppress them. In this case, they conversely became increasingly important for the interpretation. The measured signal changes continuously in time and the deviations could be expected.

The data take the form of time series and did not have the normal distribution, therefore next analysis based on the classical descriptive statistics such as an average and variance was not suitable. Representing the time series by the average value is not entirely objective, because this value of STIM signal during the stimulation can be much more different than at the end of the signal without stimulation. Just the time factor is very important in this case, because it keeps the sequence of all changes and carries useful information.

Considering the non-normal data distribution, the nonparametric statistics was used. On the basis of correlation analysis including the graphs and tables of Spearman correlation coefficients, it was apparent that the REF signals were substantially more stochastic than the STIM. We could explain this as the absolute randomness of the STIM signals was eliminated probably thanks to the one common parameter, i. e. the stimulation. Hence, the STIM signals were much more similar whether it was any regime of stimulation. The correlation analysis had rather an informative character about the relations and similarities among the signals.

For better specification, the nonparametric paired Wilcoxon test was carried out. There was tested the null hypothesis that the differences among REF and STIM signals at each horse were not significant and their medians were the same. The results showed that the signals from the reference and stimulated limbs were significantly different in each regime of stimulation in all eighty measurements except seven when the null hypothesis could not be rejected. Hard to say, what could be the real reason; if there was in fact no demonstrable effect or only some measurement error. Considering the time form of the data, the conclusion of Wilcoxon test was not sufficient because this test is based on the medians' comparison, not entirely appropriate for the stochastic signals.
The aim of further data processing was to find the relative similarities of the signals that could divide the data into the groups, and to describe the relations among and in these groups. For next analysis, the representative signals REF and STIM were obtained by the averaging of the signals of all horses within the frame of each stimulation regime. Figure 4 shows these representative signals for each stimulation regime as a result of averaging of 20 available measurements in each time step. The rising trend of STIM signals during the stimulation was more pointed up because of averaging while the disruptions in consequence of the unwanted muscle activity at some horses eliminated each other. The resulting REF signals that had not been influenced consciously during the measurement had still the stochastic character as an oscillation round its own mean value.

For the signal comparison and similarity identification, a modified algorithm of Longest Common Sub-Sequences (LCSS) was successfully used. A keynote of the algorithm is to compare two sequences so that they could be expanded in time without any rearranging the order of elements in time series whereas not all elements must be mapped [11]. The output of the algorithm was a table of distances between all pairs of sequences, so-called association matrix (Table 1). This matrix is an analogy to correlation matrix but the criterion of the relation between the sequences is the distance. The distance matrix is symmetric and the coefficient values from the interval $<0,1>$ express the strength of relation-maximum for absolutely different sequences and minimum for that identical. The distance matrix was an input for the following method of analysis.

Based on the results of Non-metric Multi Dimensional Scaling (NMDS), there is a recognizable boundary separating the reference and stimulated signals according to the dimension 1 which explains 89 $\%$ of the data variability Figure 5 (left). But the outlying location of the R11 ref far from other reference signals is not clear and the suspicion, that no difference is between the REF and STIM groups, develops also with reference to the result of the Cluster Analysis (CA) as the Figure 5 (right) shows. The explanation is brought by the analysis of the complete set of all 20 REF signals in this stimulation regime R11 using NMDS and CA again. There were found two outlying measurements with the high probability of distortion. The higher error rate is expected because the R11 regime was measured last, the horses began to be

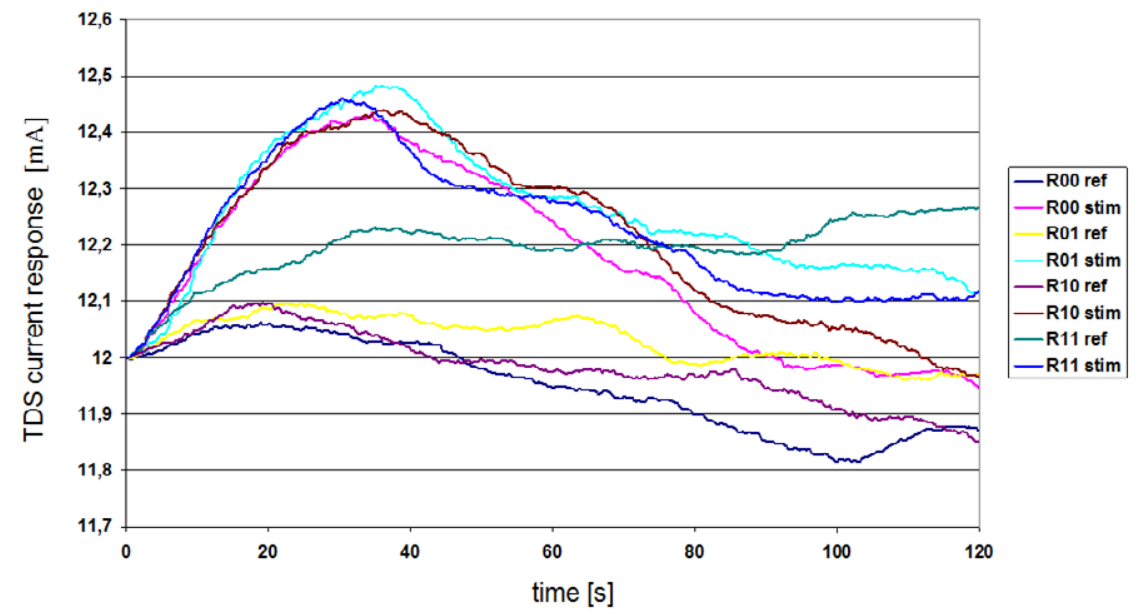

Figure 4: Representative reference (ref) and stimulated (stim) signals for each stimulation regime (R00, R01, R10, R11) as a result of averaging of 20 available measurements [5]. 
Citation: Tobolova M, Chmelar M, Provaznik I, Reznicek Z, Kabes R, et al. (2013) Testing the Effects of Micro-Pulse Stimulation on Blood Circulation Using the Thermodynamic Sensors. J Biosens Bioelectron 5: 147. doi: 10.4172/2155-6210.1000147

Page 5 of 7

\begin{tabular}{|c|c|c|c|c|c|c|c|c|}
\hline & R00 ref & R00 stim & R01 ref & R01 stim & R10 ref & R10 stim & R11 ref & R11 stim \\
\hline R00 ref & 0 & 0.9018 & 0.3710 & 0.9201 & 0.0300 & 0.9218 & 0.8586 & 0.9301 \\
\hline R00 stim & 0.9018 & 0 & 0.5008 & 0.3594 & 0.5923 & 0.0100 & 0.6639 & 0.2812 \\
\hline R01 ref & 0.3710 & 0.5008 & 0 & 0.9151 & 0.0516 & 0.6323 & 0.7837 & 0.9201 \\
\hline R01 stim & 0.9201 & 0.3594 & 0.9151 & 0 & 0.9101 & 0.2762 & 0.4493 & 0.0083 \\
\hline R10 ref & 0.0300 & 0.5923 & 0.0516 & 0.9101 & 0 & 0.8636 & 0.8037 & 0.9185 \\
\hline R10 stim & 0.9218 & 0.0100 & 0.6323 & 0.2762 & 0.8636 & 0 & 0.7022 & 0.0765 \\
\hline R11 ref & 0.8586 & 0.6639 & 0.7837 & 0.4493 & 0.8037 & 0.7022 & 0 & 0.4376 \\
\hline R11 stim & 0.9301 & 0.2812 & 0.9201 & 0.0083 & 0.9185 & 0.0765 & 0.4376 & 0 \\
\hline
\end{tabular}

Table 1: Association matrix of the distances between all signals [5].
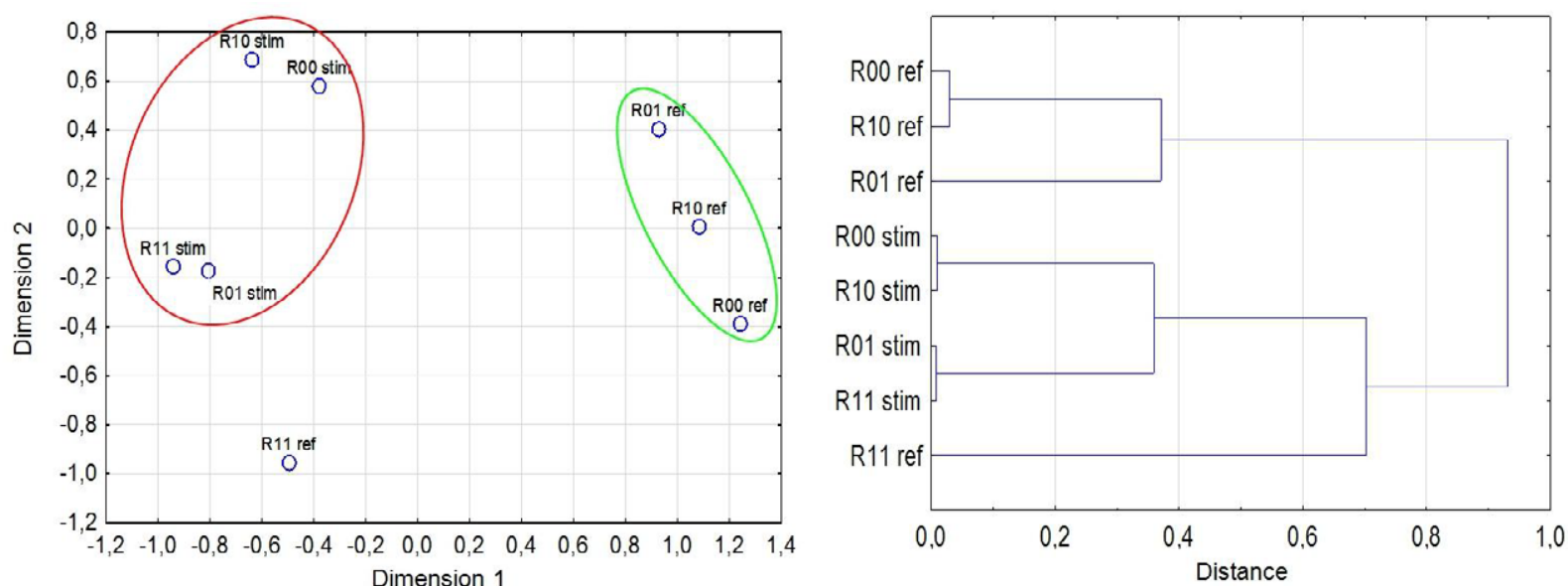

Figure 5: Left: 2D scatterplot of final configuration of the objects in the space of two dimensions as a result of non-metric multidimensional scaling with colormarked potential clusters (green-REF, red-STIM) [5].

Right: Dendrogram based on the complete-linkage clustering of the distance matrix objects [5].
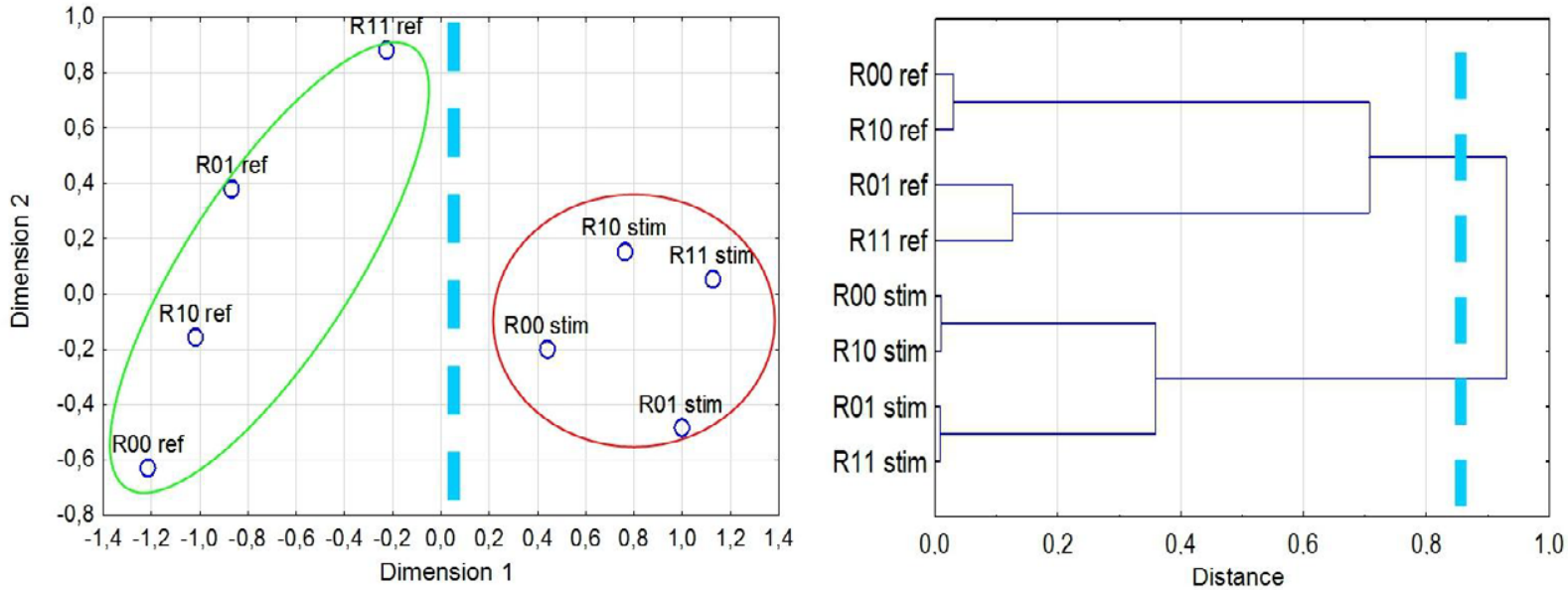

Figure 6: Left: 2D scatterplot of final configuration of the objects after the K03 ref and K08 ref elimination from the R11 regime measurement (blue line marks the possible separation of two groups in the space along the main dimension 1) [5].

Right: Dendrogram after the K03 ref and K08 ref elimination from the R11 regime (blue cut marks the possible position for the separation of the two biggest clusters REF and STIM) [5].

nervous after the long standing in one place and many more artefacts occurred compared to the previous regimes, often due to the move of the limb. The elimination of the error measurements was carried out step by step for the utmost original data preservation. All analysis steps were carried out again after each change. After the elimination of the objects $\mathrm{K} 03$ and $\mathrm{K} 08$ ref, the ability of the dimension 1 to explain the data variability increased by $2.51 \%$ compared to the original data.
The indication of the object distribution into two groups with good differentiation of the reference and stimulated signals is evident in the scaling diagram Figure 6 (left) as well as in the dendrograms Figure 6 (right).

\section{Results}

The aim of the presented statistical analysis was to answer the 
questions asked at the beginning. The methods of data processing and examination were considered carefully because the measured data are quite atypical for the classic statistic tests. The data has a form of the biological signals that change in time continuously and it cannot be described in whole range only by the simple mean value. The shape of the measured signals has an essential importance for the analysis and other analysis was based on searching the similarity among the sequences. With respect to the numerous advantages, the Longest Common Sub-Sequences algorithm was used for the signal comparison. The matrix of the distances among the REF and STIM signals in all regimes representing the whole set of data was the input for the statistical methods of the multidimensional data analysis. Based on the Non-metric Multidimensional Scaling and Cluster Analysis examining the similarity/distance of the objects, the answers to both questions can be considered.

When analysing the distance matrix of the original data, one of the reference signals (R11 ref) was classified into the group of stimulated signals. This led to the suspicions that this particular reference signal was distorted or there is no significant difference between the reference and stimulated signals, thus no demonstrable effect of the micro-pulse stimulation. It was not possible to reject neither eventuality without any detailed analysis. Two reference measurements of total twenty probably caused the distortion, and after their elimination, two wellseparated groups were established and conveniently named according to the included objects as the reference and stimulated.

The executed statistical analysis identified a significant difference between stimulated and reference signals not only by the Wilcoxon test but also the more complex methods of multidimensional analysis. This result was supported by the significant similarity of the reference, or stimulated, signals of all horses within each stimulation regime $\mathrm{Rxx}$ and even cross all regimes.

In general, the group of stimulated signals (red labelled) was more compact as compared with the reference group (green labelled). This agrees also with the correlation analysis which found multiple more relevant relations among the stimulated signals. It could be explained by the stochastic character of the reference signal which is more than stimulated signal influenced by the external and internal effects during the measurement, consequently by all eventual errors. A similar shape of the stimulated signals relates probably to the running micro-pulse stimulation.

\section{Discussion}

Based on the statistical analysis, it can be stated that the signals measured by the thermodynamic sensor at the stimulated limbs record with the high probability the thermal activity changes in consequence of the micro-pulse stimulation. When examining the depicted time series STIM, there is evident sharper growth of the amplitude for 30 seconds of the running micro-pulse stimulation. This typical response was not notable in REF signals where the micro-pulse stimulation did not proceed at all. A sine wave with a small deviation compared to the STIM signals is difficult to interpret in this moment and it calls for other measurements.

In general, the increasing tendency of the TDS signal is characteristic for the increasing thermoactivity of the sensing system, especially the thermal flow increase and warming-up. The detailed research of the exact mechanism of micro-pulse stimulation effect in the stimulated area according to the TDS signals is not realized because it is an issue of the long-term research requiring the knowledge of the veterinarian and it could be a subject of further work. Despite it and based on the micro-pulse stimulation properties and the results of previous statistical analysis, we can present a simple conception of the possible effects of the micro-pulse stimulation, limited to a description of the mechanical action of the blood vessels without relatively complicated local metabolic changes that are a scope of biochemical research.

Because of a relative low frequency of the bursts, the vasodilatation is expected as a primary effect of the micro-pulse stimulation on the blood vessels [12]. But the vasoconstriction as an effect of the used high voltage cannot be left out of consideration [13]. The burst width is another factor associated with the strength of the stimulus. Nevertheless, the resulting vasodilatation will play a major role in final consequence. Independently of the stimulus intensity, the vasoconstriction cannot persist and the vessels dilate mighty after its subsiding. True, that a reaction of each horse to the micro-pulse stimulation can be very individual and it is observable on the stimulated TDS signals. After the electrode application, a short overshoot of the signal to the lower values occurred at some horses and then it started fast increasing. This could indicate a short vasoconstriction as a psychological impact of the micro-pulse stimulation and the short paralysis, or as an initial response to the actual micro-pulse stimulation.

The purpose in the connection of the high voltage and high frequency of the carrier pulses lies in the deeper penetration into the tissue. The idea is that there are effected not only the peripheral nerve endings and small vessels but also the larger arterioles and veins. A phenomenon of vasodilatation consists in the immediate lumen extension of the blood vessel and facilitation of the blood flow that leads in the general hyperaemia of the stimulated area and close surroundings [14]. An increased blood perfusion is associated with the warming-up of the stimulated area. This is detectable by the thermodynamic sensor and reflects in the increasing tendency of the stimulated signal after the electrode application. After the electrode displacement, the growth stopped and consequently started decreasing.

\section{Conclusion}

This experiment offered the objective assignment of the physiological effect of the micro-pulse stimulation by the monitoring of the thermal changes in the stimulated limb using the thermodynamic sensors applied on the skin. Parallel to this experiment, the pilot experiment of the investigation of the possible therapeutic effect of the micro-pulse stimulation on the edema reduction of the horses' limbs with the promising result [5]. Just the edema treatment is connected with the blood circulation support, therefore these two studies are closely associated and their results complement each other.

\section{References}

1. Jayanthy AK, Sujatha N, Ramasubba Reddy M (2011) Measuring blood flow: techniques and applications-a review. International Journal of Research and Reviews in Applied Sciences 6: 203-216.

2. Rezni Cek Z, Szendiuchl, Rezni Cek M (2007) Termodynamicka senzorikaNovy pohled na monitorovani termodynamickych systemu. NOVPRESS, Brno Czech Republic.

3. Reznicek Z Sr, Szendiuch I, Reznicek M, Reznicek Z Jr (2008) Thick film double thermodynamic sensor system. $2^{\text {nd }}$ Electronics System-Integration Technology Conference, Greenwich, London, UK.

4. Tobolova M (2010) Micro-pulse stimulation and its use in physiatric treatment: Bachelor's Thesis. Brno: Faculty of Electrical Engineering and Communication, Brno University of Technology, Czech Republic.

5. Tobolova M (2012) Microstimulator: Master's Thesis. Brno: Faculty of Electrical Engineering and Communication, Brno University of Technology, Czech Republic.

6. Reznicek Z Sr, Szendiuch I, Reznicek M, Reznicek Z Jr, Tvarozek V (2007) 
Citation: Tobolova M, Chmelar M, Provaznik I, Reznicek Z, Kabes R, et al. (2013) Testing the Effects of Micro-Pulse Stimulation on Blood Circulation Using the Thermodynamic Sensors. J Biosens Bioelectron 5: 147. doi: 10.4172/2155-6210.1000147

Thick Film Sensor for Temperature Balanced Process Monitoring. Proceedings IMAPS, San Jose, USA.

7. Tvarozek V, Vavrinsky E, Reznícek Z Sr (2007) Novel approach in ratio metric technique of sensing. Journal of Electrical Engineering 58: 98-103.

8. Wissdorf H, Stashak TS, Adams OR (2008) Adams' Lahmheit bei Pferden. 4th edn, Schlutersche.

9. Hendricks BL, Dent AA (2007) International Encyclopedia of Horse Breeds. University of Oklahoma Press, Norman, USA.

10. Strupl J (1983) Chov koni. Statni zemedelske nakladatelstvi, Praha, Czech Republic.
11. Vlachos M, Hadjieleftheriou M, Gunopulos D, Keogh E (2003) Indexing multi-dimensional time-series with support for multiple distance measures. Proceeding of 9th SIGKDD, Washington, DC.

12. Vrána M, Ut’amyšev RI, Křišt'an L (1984) Elektronické přístroje pro stimulaci orgánů a tkání. 1st edn, SNTL, Praha, Czech Republic.

13. Mohr T, Akers TK, Wessman HC (1987) Effect of high voltage stimulation on blood flow in the rat hind limb. Phys Ther 67: 526-533.

14. Silbernagl S, Despopoulos A (2004) Atlas fyziologie člověka. 6th edn, Grada Publishing, Praha, Czech Republic. 